\title{
Observations on the Specificities of Extracellular Antigens of the Genera Aeromonas and Serratia
}

\author{
By P. V. LIU \\ Department of Microbiology, University of Louisville, School of Medicine, \\ Louisville, Kentucky, U.S.A.
}

(Received 2 August 1960)

\begin{abstract}
SUMMARY
Members of the genus Aeromonas produced extracellular toxic antigens which were specific for this group and antisera produced against these antigens could be used to identify these organisms. Strains of $A$. liquefaciens, A. punctata, A. hydrophila and $A$. formicans were apparently very closely related; differences which existed between them were not greater than differences found among the strains of $\boldsymbol{A}$. liquefaciens. Fresh isolates tended to be strongly haemolytic, produced extracellular toxins which were lethal to mice and elicited haemorrhagic lesions upon injection into the skin of rabbit. Old laboratory cultures tended to be much less haemolytic and less toxigenic. A. salmonicida appeared to possess extracellular antigens specific for this species but some strains of $A$. liquefaciens were found to possess this antigen.

Members of the genus Serratia were included in this study since Stevenson (1959) suggested that all Aeromonas strains are non-pigmented forms of Serratia. All strains of Serratia examined, regardless of their classification into species, were homogeneous and reacted with antiserum produced with one strain. Moreover, no cross-reaction was found between the antigens of Aeromonas and Serratia and it appeared unlikely that Aeromonas could be regarded as a non-pigmented member of Serratia. No cross-reaction was found between the extracellular antigens of these two genera and the other members of the families Enterobacteriaceae and Pseudomonadaceae and antisera produced with these antigens appeared useful in the identification of Aeromonas and Serratia.
\end{abstract}

\section{INTRODUCTION}

The genus Aeromonas is a group of Gram-negative bacilli in the family Pseudomonadaceae; they are usually motile by means of polar flagella. Unlike organisms in the genus Pseudomonas, those belonging to the genus Aeromonas ferment carbohydrate in a manner similar to that of the coliform bacteria and, therefore, the chief differences between the species of the genus Aeromonas and those in the genus Paracolobactrum are found in the arrangement of their flagella, in the less active fermentation of carbohydrates by the former, and in their pathogenicity. Although the characteristics of Aeromonas are fairly well established, no definite criterion is available at present to establish species within the genus. Snieszko (1957) used the source of isolation to differentiate three species, $A$. liquefaciens, $A$. punctata and $A$. hydrophila; and $A$. salmonicida is recognized by its pathogenicity to fish, 
particularly Salmonidae. These criteria are not adequate because similar organisms may be isolated from different sources and the virulence of an organism may be lost after prolonged cultivation on artificial media. Due to the absence of any criterion for establishing a species, several new species have been proposed without adequate comparative study with standard strains of well-established species (Pivnick \& Sabina, 1957; Stevenson, 1959).

The present study was initiated to find dependable criteria to establish the species within the genus Aeromonas. In the usual bacteriological procedures for identification of a Gram-negative bacillus of the coliform type, biochemical pattern and analyses of cellular antigens by agglutination tests are used. However, a few differences in biochemical reactions are always found even among strains of a well-known species of Aeromonas and it is difficult to determine which reactions should be taken as the differential criteria. The serological tests which use cellular antigens are frustrated by the fact that many strains of the same Aeromonas species possessed different cellular antigens. Finally, the possibility of the production of an extracellular toxin was examined because many Aeromonas strains are known to be pathogenic to cold-blooded animals, and the work of Russell (1898) suggested that the production of a toxin other than the endotoxin was possible. It was found that many fresh isolates of $A$. liquefaciens or $A$. hydrophila produced extracellular toxins, but many old laboratory cultures were relatively non-toxigenic; toxigenicity tests did not identify old laboratory strains. However, even old laboratory cultures produced non-toxic extracellular antigens which were also possessed by fresh isolates and these extracellular antigens appeared to be the most dependable criteria for identification of these Aeromonas strains. The present communication describes these findings.

\section{METHODS}

The strains of Aeromonas and Serratia used in this study are listed in Tables 1 and 2, respectively. Most of the strains of Aeromonas were obtained from Dr S. F. Snieszko (U.S. Fish and Wildlife Service, Eastern Fish Disease Laboratory, Leetown, W. Va.) and Dr R. Hugh (George Washington University, Washington, D.C.). Two strains of $A$. hydrophila isolated from sick frogs in this laboratory were also included. The strains of $\boldsymbol{A}$. margarita (Stevenson, 1958) isolated from diseased desert locusts (Schistocerca gregaria Fo̊rsk) and one strain of Pseudomonas noctuarum (Lysenko, 1958) were obtained from Dr J. P. Stevenson (Department of Zoology, University College, London). Dr Snieszko stated in a personal communication that he considered that $A$. liquefaciens, $A$. punctata and $A$. hydrophila were physiological variants of one species. $A$. margarita and $P$. noctuarum were considered by Stevenson (1959) to be identical with $S$. marcescens.

All strains of Serratia were obtained from Dr L. S. McClung (Indiana University, Bloomington, Indiana). Many of these strains were from the collection of the late Dr R. S. Breed (New York State Experiment Station, Geneva, N.Y.) and were received after his death. These strains were reclassified by Hamilton (1957) according to his criteria and, as can be seen in Table 2, the designation of the species of Serratia differed considerably according to the criteria selected for their classification.

Technique for the production of extracellular antigens. The term 'extracellular antigen' indicates all antigenic substances produced by the organisms which 
Table 1. The list of Aeromonas strains used

\begin{tabular}{|c|c|c|c|c|}
\hline Strain no. & Genus & Species & Obtained from & $\begin{array}{l}\text { Original source or } \\
\text { source of isolation }\end{array}$ \\
\hline$U-14$ & Aeromonas & liquefaciens & Dr Snieszko* & Guppy \\
\hline U-15 & Aeromonas & liquefaciens & Dr Snieszko & Guppy \\
\hline U-21 & Pseudomonas & punctata & Dr Snieszko & Dr Rucker (Seattle) \\
\hline U-23 & Pseudomonas & punctata & Dr Snieszko & Dr Lassleben (Germany) \\
\hline U-40 & Aeromonas & liquefaciens & Dr Snieszko & Dr Kluyver \\
\hline U-41 & Aeromonas & liquefaciens & Dr Snieszko & Dr Kluyver \\
\hline Canada-1958 & Aeromonas & salmonicida & Dr Snieszko & Salmon \\
\hline Leetown-1959 & Aeromonas & salmonicida & Dr Snieszko & Salmon \\
\hline A-H-1 & Aeromonas & hydrophila & This laboratory & Frog \\
\hline A-H-2 & Aeromonas & hydrophila & This laboratory & Frog \\
\hline 251 & Aeromonas & hydrophila & Dr Hugh $\uparrow$ & ATCC $7965 \&$ \\
\hline 513 & Aeromonas & formicans & Dr Hugh & $\begin{array}{l}\text { ATCC 13136. Pivnick's } \\
\text { strain } 258\end{array}$ \\
\hline 514 & Aeromonas & formicans & Dr Hugh & $\begin{array}{l}\text { ATCC 13137. Crawford's } \\
\text { Pacific Grove strain }\end{array}$ \\
\hline 618 & Aeromonas & & Dr Hugh & Water of Potomac River \\
\hline 944, & Aeromonas & hydrophila & Dr Hugh & $\begin{array}{l}\text { Murray's 6, 1:A-1 from } \\
\text { trout }\end{array}$ \\
\hline 829 & Aeromonas & sp. & Dr Hugh & Water from Rock Creek \\
\hline $832-5$ & Aeromonas & sp. & Dr Hugh & Minced beef \\
\hline SL 10 & Aeromonas & margarita & Dr Stevenson $\ddagger$ & Desert locust \\
\hline SL 11 & Aeromonas & margarita & Dr Stevenson & Desert locust \\
\hline SM 006 & Pseudomonas & noctuarum & Dr Stevenson & $\begin{array}{l}\text { Dr Lysenko (Czecho- } \\
\text { slovakia) }\end{array}$ \\
\hline
\end{tabular}

* U.S. Fish and Wildlife Service, Eastern Fish Disease Laboratory, Leetown, W. Va.

$\dagger$ George Washington University, Washington D.C.

¥ Department of Zoology, University College, London

$\$$ American Type Culture Collection.

Table 2. The list of Serratia strains used

Strain no. assigned by Hamilton*

$\begin{array}{cl}8 & \text { S. } \text { indica } \\ 11 & \text { S. indica } \\ 16 a & \text { S. marcescens } \\ 16 b & \text { S. marcescens } \\ 59 a & \text { S. plymuthica } \\ 60 & \text { S. marinorubra } \\ 61 & \text { S. marcescens } \\ 62 & \text { S. marinorubra } \\ 63 & \text { S. marcescens } \\ 113 & \text { S. kiliensis } \\ 139 & \text { S. plymuthica } \\ 146 & \text { S. plymuthica } \\ 151 & \text { S. kiliensis } \\ 230 a & \text { S. marcescens }\end{array}$

Strain no.

Name as received as received by by McClung* McClung

Original source

\section{S. marcescens}

53A

S. marcescens

53 B

S. rubra

S. rubra

Serratia sp.

Serratia sp.

Serratia sp.

Serratia sp.

Serratia sp.

Erythrobacillus sp.

S. plymuthica

S. plymuthica

S. marcescens

Serratia sp.
U. of Wisconsin

U. of Wisconsin

Ohio State Univ.

Ohio State Univ.

$\mathrm{CDC} \dagger$

CDC

CDC

CDC

CDC

Breed collection

Breed collection

Breed collection

Breed collection

Beth Israel Hosp.

N.Y.

* Indiana University, Bloomington, Indiana.

† Communicable Disease Center, U.S. Public Health Service.

One strain of Serratia marcescens from the stock cultures of this department was included in this study. 
could be separated readily from the cells. This antigen was produced with the cellophan plate technique already described ( $\mathrm{Liu}, 1957$ ). It consisted essentially in cutting a sheet of cellophan to fit the bottom of a Petri dish, sterilizing it by steam under pressure and placing it in the dish after the appropriate medium was poured and solidified. After the cellophan sheet was moistened, the wrinkles were carefully stretched out. About two drops of broth culture of the organism were placed on the cellophan and spread over the whole plate with a sterile cotton swab. The plate was incubated aerobically at the optimal temperature of each organism, which was $24^{\circ}$ for Aeromonas salmonicida and $\mathbf{2 8}^{\circ}$ for all other Aeromonas and all Serratia species. The medium used for this purpose was the Trypticase-soy agar (Baltimore Biological Laboratories) enriched with $0.5 \%$ glycerol. At the end of the incubation period, which was usually about $48 \mathrm{hr}, 3 \mathrm{ml}$. sterile saline was used to wash off the growth which was centrifuged for $60 \mathrm{~min}$. at $2000 \mathrm{~g}$ to remove the bacterial cells. The supernatant fluid containing the extracellular antigen was Seitz-filtered and preserved by the addition of enough thiomersal (Merthiolate, Lilly) to make the final concentration of $1 / 5000$.

Production of antisera to the extracellular antigen. Aeromonas strains U-15, U-40, Canada-1958 and five strains of Serratia representing five species $(8,16 a$, $59 a, 60$ and 113) were used for the production of antisera. Strain U-15 was a relatively recent isolate of $A$. liquefaciens and it produced strong haemolysis on blood agar. U-40 on the contrary was an old laboratory culture which was used by the late Dr A. J. Kluyver as a typical strain of $A$. liquefaciens. It was almost rough in its colonial morphology and the extracellular antigens of this strain were found to be the least toxic among those produced by various strains of $\boldsymbol{A}$. liquefaciens. It was used, therefore, as a representative of $A$. liquefaciens which became rough and non-toxigenic after a prolonged culturing on artificial media. The two strains of A. salmonicida did not vary much in their toxigenicity and the strain Canada-1958 was used because it was much more chromogenic and appeared to be more typical of this species.

After sterility tests of the extracellular antigen, it was mixed with an equal amount of Freund's incomplete adjuvant (a mixture of Arlacel and Bayol in the ratio of 1:5/8.5) and injected subcutaneously into rabbits at weekly intervals; $0.2 \mathrm{ml}$. was the dose for all antigens in the first week and $0.5 \mathrm{ml}$. in the second week. In the cases of U-40, Canada-1958 and all Serratia strains, 0.5 and $1.0 \mathrm{ml}$. of antigen without the adjuvant were injected intravenously in the 3rd and 4th weeks. The antigens of U-15 were so toxic that $0.2 \mathrm{ml}$. injected intravenously killed a rabbit within $8 \mathrm{hr}$. The preparation was diluted, therefore, ten times for intravenous injection. Beginning in the $3 \mathrm{rd}$ week, $0.2,0.5,1.0$ and $2.0 \mathrm{ml}$. of the diluted antigen were injected intravenously at 3 -day intervals. The sera were obtained 1 week after the last injection and preserved with thiomersal.

Identification tests. The technique used for the identification of these organisms with antisera was essentially that devised by Elek (1948) for the in vitro toxigenicity test of Corynebacterium diphtheriae. The medium used for this purpose contained Tryptose (Difco) $0.1 \%$, Casamino acids (Difco) $0.3 \%$, glycerol $0.5 \%, \mathrm{Na}_{2} \mathrm{HPO}_{4}$ $0.8 \%, \mathrm{NaCl} 0.2 \%$ and Bacto-agar $1.2 \%(\mathrm{pH} 7 \cdot 4)$. The paper strip contained $0.15 \mathrm{ml}$. undiluted antiserum. Before inoculation of the organisms the plate was incubated overnight at $37^{\circ}$ to ensure a dry surface. The inocula were always taken from fresh 
cultures on blood agar. The plates which contained Aeromonas salmonicida were incubated at $24^{\circ}$ because these organisms did not grow well at a higher temperature. All other plates were incubated at $28^{\circ}$.

\section{RESULTS}

Intradermal lesions produced by the extracellular antigens of Aeromonas and Serratia

The lesions produced by the extracellular antigens of various species of Aeromonas in the skin of rabbit are shown in Pl. 1, fig. 1. The extracellular antigens of U-15, A-H-1 and 514 contained toxins which produced haemorrhagic lesions. This was characteristic of most of the fresh isolates of motile Aeromonas strains, regardless of species. The haemorrhagic lesions appeared within $5 \mathrm{~min}$. of the intracutaneous injection of $0.1 \mathrm{ml}$. of the crude preparation and were similar to the lesions produced by the toxin of Pseudomonas pseudomallei described by Nigg, Heckley \& Colling (1955). The lesions reached maximum development within $3 \mathrm{hr}$. There were a few strains of the motile Aeromonas (such as U-40 and U-21 and mostly old laboratory cultures) which did not produce the haemorrhagic toxin (Pl. 1, fig. 1). It is possible that these strains produced toxin when they were first isolated but had lost this ability after prolonged cultivation on artificial media. The two strains of $\boldsymbol{A}$. salmonicida did not produce the haemorrhagic toxin; since both were relatively recent isolates, the lack of haemorrhagic toxin in their extracellular antigens probably indicates a characteristic of the species.

The lesions produced by the extracellular antigens of Serratia are shown in Pl. 1, fig. 2. Fifteen strains of Serratia representing five species were tested. Preparations from some strains produced only abscesses and redness similar to the lesions produced by the endotoxin of Gram-negative bacteria, but preparations from other strains contained toxin which produced haemorrhagic and necrotic lesions. There was no correlation between pigment production and the production of these toxins, or between the type of lesion produced by a strain and its classification into species.

\section{Neutralization of the toxic effects by antiserum}

The haemorrhagic toxin of Aeromonas hydrophila, A. punctata and A. formicans could be neutralized by the antiserum to one strain of $A$. liquefaciens (U-15) as shown in Pl. 1, fig. 3. Quantities of serial two-fold dilutions of antiserum in saline were added to an equal amount of the toxin of $A$. hydrophila and $A$. formicans and the mixtures incubated at 37 for $1 \mathrm{hr}$; $0 \cdot 1 \mathrm{ml}$. each of the mixtures was injected intracutaneously into the rabbit. It can be seen in Pl. 1, fig. 3, that the toxins of both $A$. hydrophila and $A$. formicans were neutralized by the antiserum of $A$. liquefaciens.

Attempts to demonstrate neutralization by protection tests in mice were not successful since the lethal effect of U-15 toxin was not completely neutralized by its homologous antiserum.

\section{Identification tests with Aeromonas species}

In Pl. 2, figs. 4 and 5, are shown some of the results obtained in the agar diffusion identification test. Positive results could be noted readily by the formation near the paper strip of the so-called 'arrow head figure' which was due to precipita- 
tion of antigens with their homologous antibodies. Most of the motile strains of Aeromonas used in this study were found to react readily with U-15 antiserum: There were a few strains of $A$. liquefaciens (including U-40) which did not react with U-15 antiserum and instead, they were found to react with Canada-1958 (A. salmonicida) antiserum, as shown in Fig. 5.

\section{Identification tests with Serratia species}

In their extracellular antigens the members of the genus Serratia appeared to be homogeneous. All strains of Serratia used in this study, regardless of their pigmentation and designation into species, gave a reaction with antiserum produced with one strain of Serratia. None of the Aeromonas strains except the two of Aeromonas margarita and the strain of Pseudomonas noctuarum showed reaction with antiserum of the Serratia group.

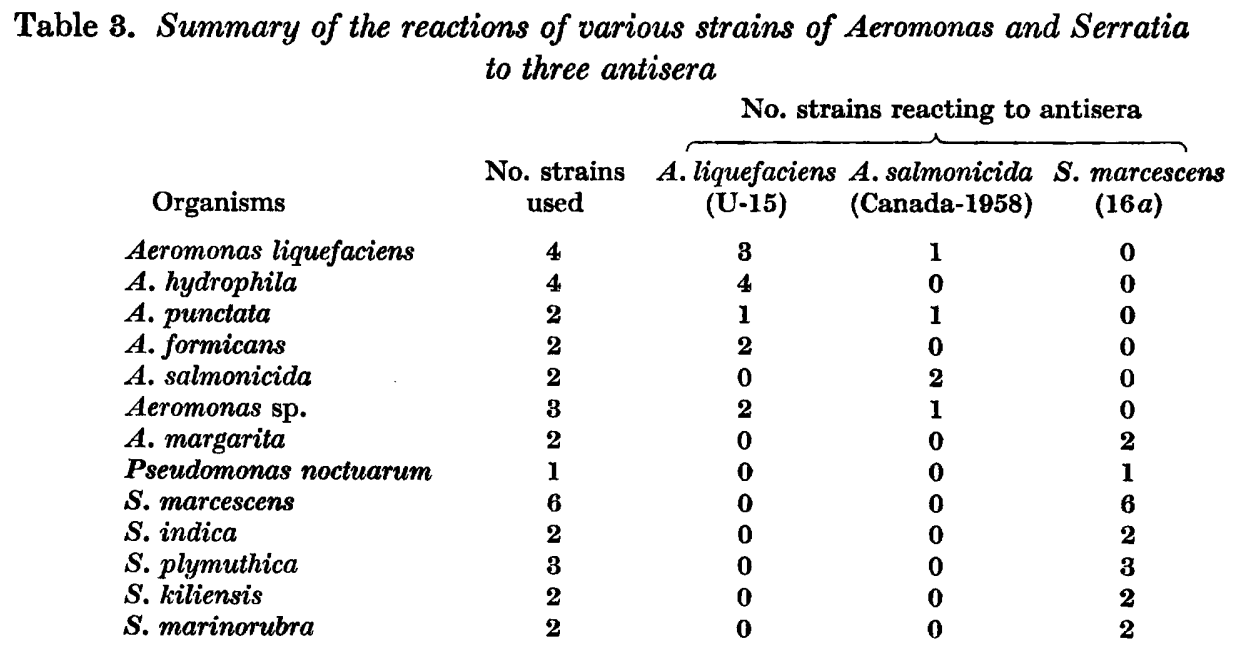

The results of these identification tests are summarized in Table 3 . It should be noted that most of the motile strains of Aeromonas belonging to the species $A$. hydrophila, $A$. punctata and $A$. formicans reacted readily with antiserum of $A$. liquefaciens and a few of them reacted with antiserum of $A$. salmonicida. The two strains of $\boldsymbol{A}$. margarita and one strain of Pseudomonas noctuarum reacted readily with antiserum of Serratia marcescens and therefore these organisms appeared to be merely non-pigmented forms of Serratia, as suggested by Stevenson (1959). However, the generalization that all Aeromonas strains are non-pigmented forms of Serratia could not be substantiated because none of the other strains of Aeromonas showed any cross-reaction with antisera of the Serratia group.

No cross-reaction was observed between Aeromonas, Serratia and other genera of the families Enterobacteriaceae and Pseudomonadaceae; therefore antisera prepared with extracellular antigens of strains of Aeromonas and Serratia appeared to be useful in the identification of these genera. 


\section{DISCUSSION}

The evidence presented in this paper indicates that the organisms belonging to the genera Aeromonas and Serratia produce extracellular antigens specific for each genus and that antisera produced with these antigens can be used to identify organisms belonging to these genera. Members of the genus Aeromonas are known to be pathogenic to various cold-blooded animals, such as frogs and fish (Emerson $\&$ Norris, 1905), and infection in man has been reported (Caselitz \& Buck, 1958). Members of the genus Serratia are not generally considered to be animal pathogens but infections with members of this genus have been reported (Wheat, Zuckerman \& Rantz, 1951; Vernon \& Hepler, 1954). The production of toxic extracellular antigens by these organisms may play some role in the pathogenesis of these organisms.

Strains of Aeromonas which have been determined as $A$. liquefaciens, $A$. hydrophila, $A$. punctata and $A$. formicans are apparently very closely related organisms separated into different species on the basis of the source of isolation and a few biochemical reactions. The differences between these species were not greater than the differences found between the various strains of $A$. liquefaciens. The fresh isolates of this group tended to be strongly haemolytic, produced extracellular toxins and various extracellular enzymes, and most of their extracellular antigens reacted with antiserum against toxigenic strains of $A$. liquefaciens, such as U-15. $A$. salmonicida is a different organism as can be seen by its lower optimal temperature of growth, production of pigment and non-motility. However, the crossreactions between this species and some strains of motile Aeromonas indicate that all strains of Aeromonas are closely related, and the absence of cross-reaction with other Gram-negative bacilli indicates that organisms belonging to the genus Aeromonas are a distinct group.

Members of the genus Serratia were included in this study at a later stage because Stevenson (1959) suggested that Aeromonas species are non-pigmented members of the genus Serratia. Complete absence of cross-reaction between strains of these two genera would seem to exclude the possibility that Aeromonas can be regarded as non-pigmented forms of Serratia. Stevenson (1959) drew his conclusion because of the similarity of $\boldsymbol{A}$. margarita and $S$. marcescens and did not use typical strains of Aeromonas for comparative study. Since both strains of the so-called $A$. margarita used in this study reacted readily with antisera of the Serratia group and not with those of the Aeromonas group it was obvious that these two strains were non-pigmented members of Serratia and not Aeromonas. The results of the present study indicated that members of the genus Serratia were quite closely related in spite of the differences in pigment production and a few biochemical reactions which were used to differentiate them into species. Antisera produced against extracellular antigens of one strain of Serratia will identify practically all strains of Serratia regardless of their classification into species, which, according to Breed (1957), depends on minor differences such as solubility of the pigments and pellicle formation, characters that are variable. Inspection of Table 2 will show how different and confusing the classification of this group can be, depending on the criteria preferred by different workers. In view of the cross-reactions between the antigens of various strains of Serratia, classified into different species, it is doubtful whether any good can be 
achieved by such a classification. A comparison between the classification of Corynebacterium diphtheriae and that of the Serratia species will serve to elucidate this point. The species $C$. diphtheriae has been partially established on the basis of its production of an exotoxin. The species has a certain biochemical pattern by which organisms belonging to it can be identified. Strains of the species ferment glucose, maltose and dextrin but not sucrose or lactose. The species $C$. diphtheriae is further divided into gravis, mitis and intermedius varieties by their colonial morphology and a few other biological characteristics. For example, the gravis variety tends to form a pellicle on the surface of broth and to ferment starch. Neither mitis nor intermedius varieties ferment starch and they do not usually form a pellicle. Mitis is usually haemolytic but intermedius is not. In the case of Serratia the characteristic of pellicle formation was used by Breed (1957) to separate $S$. indica from $S$. marcescens. If this criterion were to be taken for the classification of corynebacteria, a pellicle-forming strain of $C$. diphtheriae of the gravis variety would be assigned the rank of an independent species. Since some gravis strains do not form a pellicle but do ferment starch, another species would have to be created for this kind of strain. Solubility of pigments was used by Breed (1957) as a criterion in the separation of Serratia species, but pigment production may be lost by some strains. The so-called Aeromonas margarita is an excellent example of a non-pigmented form which is difficult to identify if pigment production is used as a distinguishing criterion.

The differences that existed between the various strains of Serratia tested, although classified into different species, did not appear to be greater than the differences that can be found among strains of other bacteria placed in a single species. The group as a whole could be placed in a single species and different subspecies night be recognized by the characteristics, such as pigment formation and the production of gas or acetylmethylcarbinol, which are not common to all strains.

The extracellular antigens produced by organisms belonging to the family Pseudomonadaceae have received very little, if any, attention in the past. This is due undoubtedly to the fact that the procedures and techniques used at present for the identification of Gram-negative bacilli are those developed primarily for the identification and classification of members of the family Enterobacteriaceae. The enteric bacteria are not active producers of extracellular enzymes or toxins and, therefore, the classification and identification of these organisms depend primarily on their biochemical reactions and cellular antigens. The members of the family Pseudomonadaceae and the genus Serratia (although it is included by Breed \& Murray (1957) in the family Enterobacteriaceae) are much more active producers of extracellular antigens and some of these antigens, in being heat labile, have some characters of exotoxins. Both from the viewpoint of identification of the organisms and the understanding of their role in pathogenesis, these antigens deserve more attention than they have been accorded hitherto.

This work was done during the tenure of a senior research fellowship (SF-259) from the National Institute of Health, U.S. Public Health Service. 
Breed, R. S. (1957). In Bergey's Manual of Determinative Bacteriology, 7th ed. p. 359. Baltimore: Williams and Wilkins Co.

Breed, R. S. \& Murray, E. G. D. (1957). In Bergey's Manual of Determinative Bacterio$\log y, 7$ th ed. p. 332. Baltimore: Williams and Williams Co.

Caselitz, F. H. \& Buck, A. A. (1958). Biologische Studien an Mikroorganismen des Genus Aeromonas. Zbl. Bakt. 1. Orig. 173, 407.

Elek, S. D. (1948). The recognition of toxicogenic bacterial strains in vitro. Brit. med. $J$. i, 493.

Emerson, H. \& Norris, C. (1905). 'Red-leg'-an infection disease of frogs. J. exp. Med. $7,32$.

Hamilton, R. D. (1957). A critical study of the genus Serratia. Thesis. Indiana University, Bloomington, Indiana.

LrU, P. V. (1957). Survey of hemolysin production among species of pseudomonads. J. Bact. 74, 718.

Lysenko, O. (1958). Contribution to the taxonomy of Coccobacillus acridiorum d'Herelle. Folia biol., Prague, 6, 342.

Nigg, C., Heckley, R. J. \& Colling, M. (1955). Toxins produced by Malleomyces pseudomallei. Proc. Soc. exp. Biol., N.Y. 89, 17.

Pivnick, H. \& Sabina, L. R. (1957). Studies of Aeromonas formicans Crawford comb. nov. from soluble oil emulsions. J. Bact. 73, 247.

Russell, F. H. (1898). An epidemic septicemic disease among frogs due to the Bacillus hydrophilus fuscus. J. Amer. med. Ass. 30, 1442.

SnIEszko, S. F. (1957). In Bergey's Manual of Determinative Bacteriology, 7th ed., p. 189. Baltimore. Williams and Wilkins Co.

Stevenson, J. P. (1958). A study of the microbiology of an insect infection. Thesis. University of London.

Stevenson, J. P. (1959). A note on the genus Aeromonas. J. gen. Microbiol. 21, 366.

Vernon, R. G. \& Hepler, O. E. (1954). Chromogenic bacteria with a case report of a fatal infection caused by Serratia marcescens. Quart. Bull. Northw. Univ. med. Sch. 28, 366.

Wheat, R. P., Zuckerman, A. \& Rantz, L. A. (1951). Infection due to chromobacteria; report of eleven cases. Arch. intern. Med. 88, 461 .

\section{EXPLANATION OF PLATES}

Plate 1

All photographs in these three figures were taken $18 \mathrm{hr}$. after the injections.

Fig. 1. The lesions produced by the extracellular antigens of various species of Aeromonas in the skin of rabbit. They are (from left to right, upper row); 514 (Crawford's Pacific Grove strain of A. formicans), A-H-1 (A. hydrophila), U-15 (A. liquefaciens), (left to right, lower row); Canada-1958 (A. salmonicida), U-21 (A. punctata) and U-40 (A. liquefaciens).

Fig. 2. The lesions produced by the extracellular antigens of fifteen strains of Serratia representing five species. The antigens of some strains contained haemorrhagic and necrotic toxins while those of the other strains did not. There was no correlation between pigment production, species designation of a strain and its toxin production.

Fig. 3. Neutralization of the haemorrhagic toxins of $A$. formicans and A. hydrophila by A. liquefaciens antiserum. The upper row are the toxin of 514 (A. formicans) added to an equal amount of twofold dilutions of an antiserum of U-15 (A. liquefaciens), starting from $\frac{1}{4}$ from left side. $0.1 \mathrm{ml}$. of the mixtures was injected intracutaneously. The lower row are the toxin of A-H-1 (A. hydrophila) added to the same dilution of the same antiserum. The corresponding dilutions of the antiserum are connected by a black line. The control toxins of both species are connected by double lines. 


\section{Plate 2}

Fig. 4. Five strains of Aeromonas tested with antiserum of U-15 (A. liquefaciens). They are (left side of the paper strip from top to bottom): U-15, U-21 (A. punctata), U-40 (A. liquefaciens); (right side of paper strip from top to bottom), Canada-1958 and Leetown-1959 (both A. salmonicida). Note that only the strain U-15 is reacting to this serum (the so-called arrow head figure).

Fig. 5. The same five strains in the same arrangement as shown in Fig. 4 tested with an antiserum of Canada-1958. Note that both strains of $A$. salmonicida are showing strong reactions. The two strains of motile Aeromonas (U-21 and U-40) are also showing some reactions, although they are not as strong as those of $A$. salmonicida.

The two photographs in Figs. 4 and 5 were taken after 5 days incubation at $24 \%$ 
Journal of General Microbiology, Vol. 24, No. 1

Plate 1
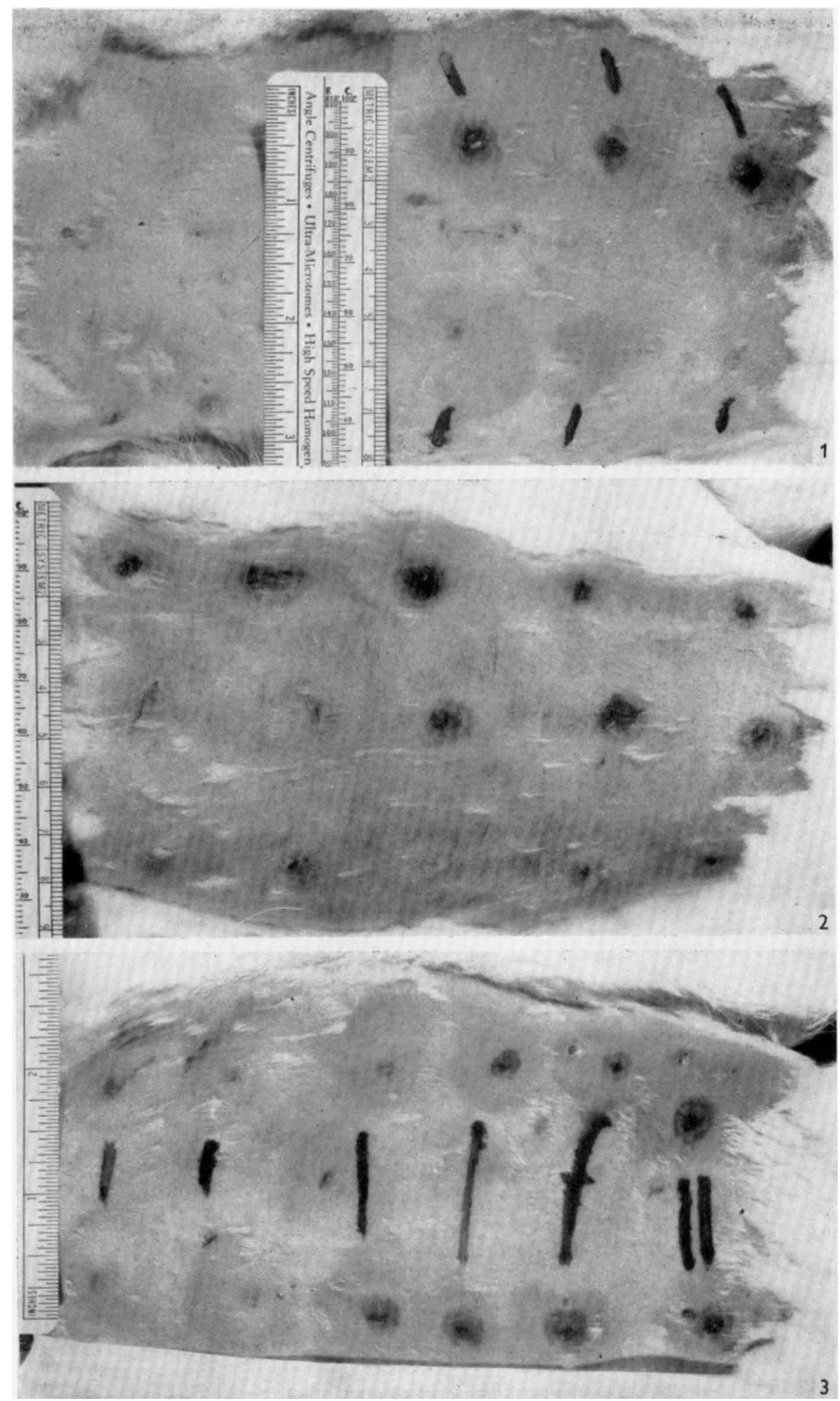

P. V. LIU

(Facing p. 154) 

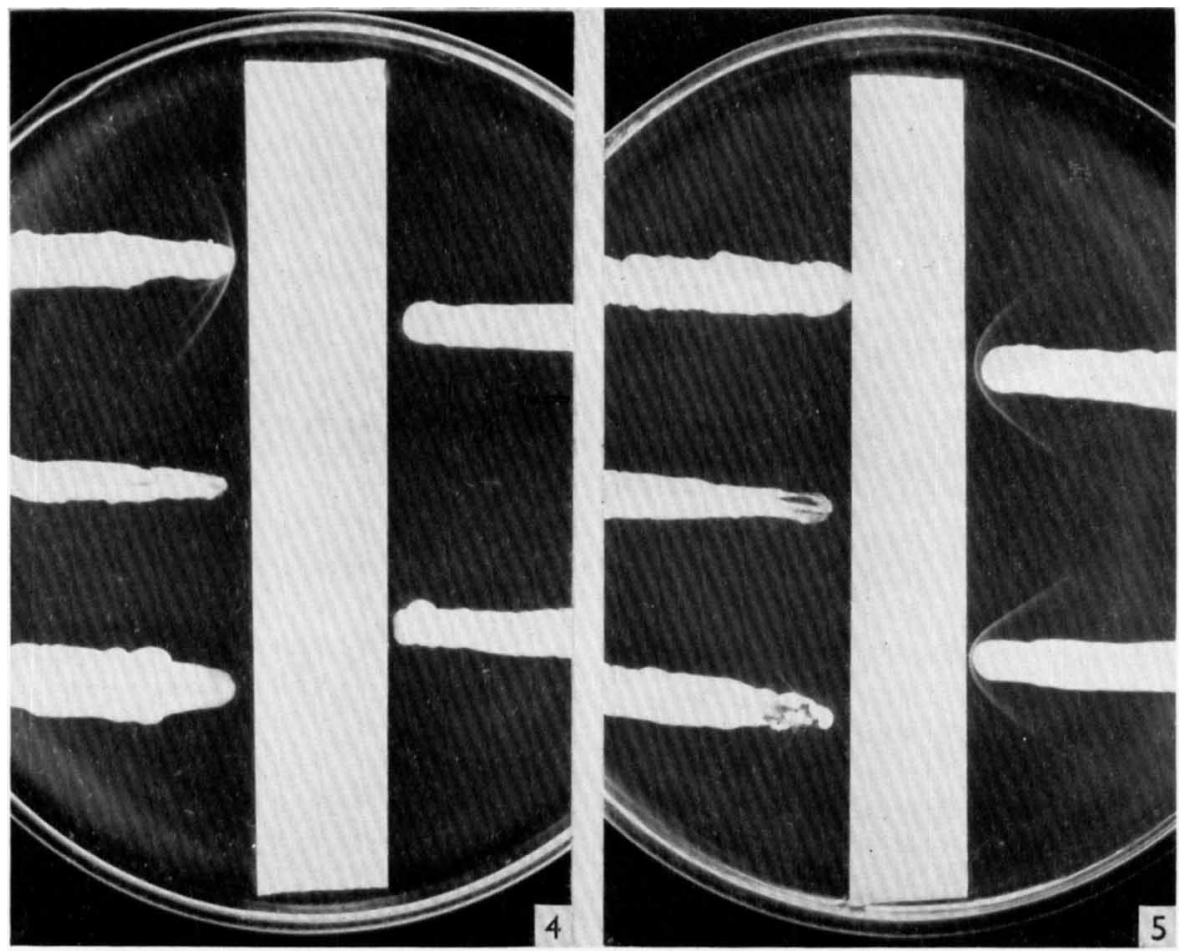